\title{
Mechanical design, analysis and testing of a large-range compliant microgripper
}

\author{
Yilin Liu and Qingsong Xu \\ Department of Electromechanical Engineering, Faculty of Science and Technology, University of Macau, \\ Avenida da Universidade, Taipa, Macau, China \\ Correspondence to: Qingsong Xu (qsxu@umac.mo)
}

Received: 15 December 2015 - Revised: 9 March 2016 - Accepted: 24 March 2016 - Published: 12 April 2016

\begin{abstract}
This paper presents the mechanical design, analysis, fabrication, and testing procedures of a new large-range microgripper which is based on a flexible hinge structure. The uniqueness of the gripper is that the gripper arms not only provide large gripping range but also deliver approximately rectilinear movement as the displacement in nonworking direction is extremely small. The large gripping range is enabled by a mechanism design based on dual-stage flexure amplifier to magnify the stroke of piezoelectric actuator. The first-stage amplifier is a modified version of the Scott Russell (SR) mechanism and the second-stage amplifier contains a parallel mechanism. The displacement amplification ratio of the modified SR mechanism in the gripper has been enlarged to 3.56 times of the conventional design. Analytical static models of the gripper mechanism are developed and validated through finite-element analysis (FEA) simulation. Results show that the gripping range is over $720 \mu \mathrm{m}$ with a resonant frequency of $70.7 \mathrm{~Hz}$ and negligible displacement in nonworking direction. The total amplification ratio of the input displacement is 16.13. Moreover, a prototype of the gripper is developed by using aluminium 7075 for experimental testing. Experimental results validate the analytical model and FEA simulation results. The proposed microgripper can be employed in various microassembly applications such as pick-and-place of optical fibre.
\end{abstract}

\section{Introduction}

With the rapid development in micro-electromechanical systems (MEMS) and precision engineering, extensive research on automatic micromanipulation and microassembly technology has been carried out. As one of the usual manipulators, microgripper is an essential device in the micro/nanomanipulation (Han et al., 2006; Lamers et al., 2015). A typical microgripper consists of the actuator and guiding mechanism. Recently, researchers have created different kinds of the grippers, such as the adhesion-type and mechanical-type grippers (Arai and Fukuda, 1997; Tanikawa et al., 1996). In some applications, the gripper operating performance is limited by the gripping range. So, many amplification devices, such as bridge-type amplifiers, have been used to enhance the output displacement ( $\mathrm{Ai}$ and $\mathrm{Xu}, 2014$ ). These studies are of practical significance for the realization of the microassembly technology.
To realize an ultrahigh precision displacement, the flexure mechanisms have been widely applied (Dunning et al., 2011; Merriam et al., 2013; Tian et al., 2015). In addition, lots of micro-/nanogrippers have been driven using smart actuators, e.g., piezoelectric stack actuators, which have been applied to biological cell manipulation and MEMS (Bergander et al., 2003; Götze and Pagel, 2007). Piezoelectric (PZT) actuator has many advantages, such as high resolution, faster response speed, larger force output and low power consumption (Khadraoui et al., 2014; Xu, 2013; Zhang et al., 2015). But it has limited output displacement. Hence, the output is not enough for the grasping task in many cases. This issue can be overcome by resorting to displacement amplification mechanisms.

Concerning the structure design of a gripper, various designs have been devised by using compliant mechanisms. The output motion of the compliant mechanisms is produced by using the elastic deformation of the material. Hence, com- 
pliant mechanisms can provide some advantages, such as friction free and lubrication free. However, the output motions of majority of existing microgrippers are generated in a rotary way. Hence, if the gripper tips come to contact with the target object which has a curved shape, the reaction force will push out the object. Scott Russell (SR) mechanism has been used to design micromanipulation systems previously (Hwang et al., 2011; Chang and Du, 1998; Ha et al., 2006). SR mechanism has a simple structure and it can provide rectilinear output motion. But its disadvantage is also clearly. That is, its amplification ratio is not large enough. This means that the SR mechanism may reduce the output displacement of the actuator. However, the output of a PZT actuator in a micromanipulator is small already. Hence, one motivation of this work is to overcome this problem by devising an improved SR mechanism.

Motivated by the aforementioned review, this paper presents the mechanical design, fabrication, and testing procedures of a new large-range compliant microgripper, which is based on flexure-hinge structure. The structure of the gripper is illustrated in Fig. 1. The main advantage of the gripper is that the gripper arms not only provide large-range output displacement but also produce approximately rectilinear movement, because the nonworking directional displacement is extremely small. In this design, the gripper is actuated by only one PZT actuator which is fixed in the middle of the device. The actuator delivers the resolution of $0.45 \mathrm{~nm}$ (openloop) and its output stroke is $45 \mu \mathrm{m}$. A two-sided dual-stage flexure amplifier is designed to magnify the stroke of PZT actuator twice. The first-stage amplifier is a modified version of the SR mechanism, which is placed in a hypotenuse position. The monolithic components are connected by using circular notch hinges (Merken et al., 2004). With this SR mechanism, the gripper can provide both amplified and approximately rectilinear output. The amplification ratio of the modified SR mechanism has been enlarged to 3.56 times of the existing design. The second-stage amplifier is a parallelogram which can resist the nonworking directional deformation. This amplifier has been used to design a metamorphic gripper for microassembly in the previous work (Bruzzone and Bozzini, 2010).

In this work, the total amplification ratio for the input displacement is 16 and the achieved gripping range is $720 \mu \mathrm{m}$. For experimental verification, a mesoscale prototype is developed by using a PZT actuator. The actuator has a length of $68 \mathrm{~mm}$. The gripper size can be reduced by changing the actuation approach. Detailed design procedures are presented in the following parts of the paper.

\section{Mechanism design and modeling}

The mechanism design of the microgripper is presented in this section. It involves the guiding mechanism design, the

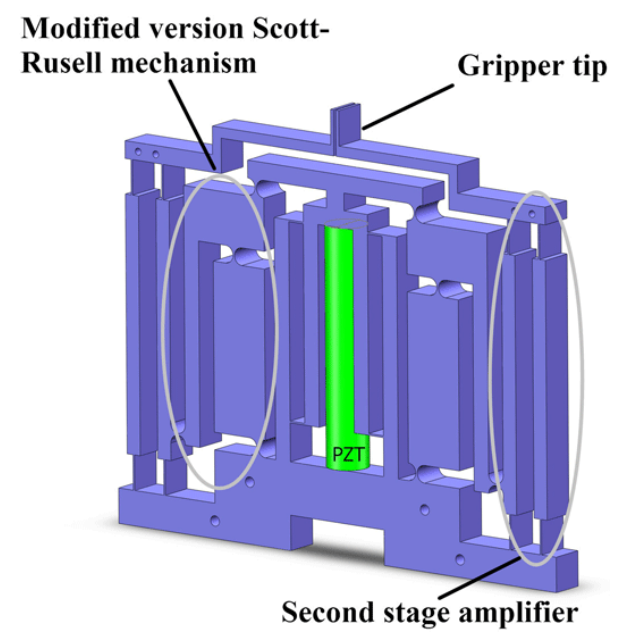

Figure 1. The mechanical structure of the designed microgripper.

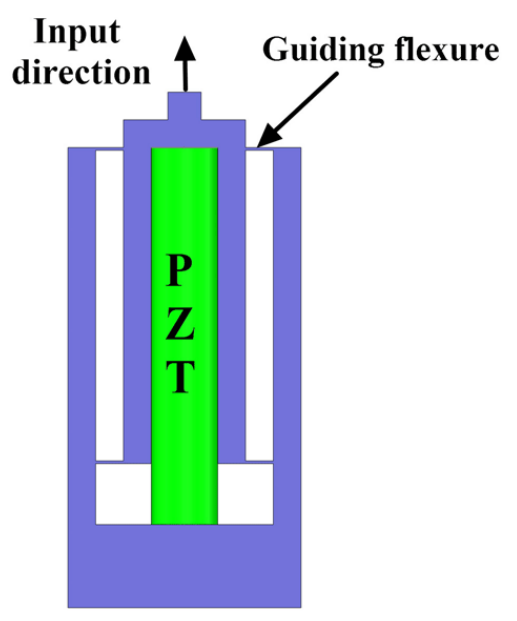

Figure 2. CAD model of guiding mechanism.

first-stage and second-stage amplifier design, and dynamics modeling of the gripper.

\subsection{Guiding mechanism design}

For driving the gripper, the actuation displacement is guided by four leaf flexures. The computer-aided design (CAD) model of the guiding mechanism is depicted in Fig. 2. When the output force of the PZT actuator is exerted on this guiding mechanism, the guiding flexures produce a purely translational movement. Each leaf flexure behaviors like a fixedguided beam. In view of the boundary conditions of the two ends of the beam, i.e., the rotation angle of the ends is zero and the deformation of the guided end is $\delta_{x}$, the following 
equations are generated.

$$
\begin{aligned}
0 & =\frac{F l^{2}}{2 E I}-\frac{M l}{E I} \\
\delta_{x} & =\frac{F l^{3}}{2 E I}-\frac{M l^{2}}{E I},
\end{aligned}
$$

where $l$ is the length of the leaf flexure, $F$ denotes the force, $M$ represents the moment, and $E$ is elastic modulus of the material.

The moment of inertia can be calculated as follows.

$$
I_{\mathrm{b}}=\frac{m_{\mathrm{b}} l^{2}}{12},
$$

where $m_{\mathrm{b}}$ is the mass of the leaf flexure.

The solutions to the above two Eqs. (1) and (2) are shown below.

$$
\begin{aligned}
M & =\frac{F l}{2} \\
\delta_{x} & =\frac{F l^{3}}{12 E I}
\end{aligned}
$$

From the kinetic energy equation and the kinematic relationship of the mechanism, the expression of the equivalent mass can be derived:

$M_{1}=m_{\mathrm{a}}\left(\frac{1}{4}\right)^{2}+4 m_{\mathrm{b}}\left(\frac{1}{8}\right)^{2}+4 I_{\mathrm{b}} \frac{1}{(8 l)^{2}}$,

where $m_{\mathrm{a}}$ is the mass without the leaf flexure, as shown in Fig. 6 later.

The potential energy of the guiding flexures mechanism is obtained as follows.

$V=4\left(\frac{1}{2} K_{\mathrm{s}} \theta_{z}^{2}\right)=\frac{2 K_{\mathrm{s}} q^{2}}{(8 l)^{2}}$,

where $K_{\mathrm{S}}$ is the stiffness, $\theta_{z}$ is the rotational angle, and $q$ denotes the deformation at the end of each beam. The details can be referred to the previous work $(\mathrm{Xu}, 2013)$.

For the presented structure, the stiffness $K_{\mathrm{s}}$ is obtained below (Xu, 2013):

$K_{\mathrm{s}}=2 \gamma K_{\theta} \frac{E I}{l}$,

where $\gamma=0.85$ and $K_{\theta}=2.6686$.

Hence, the natural frequency of the guiding mechanism is calculated as shown below.

$f=\sqrt{\frac{K}{M_{1}}}$,

where

$K=\frac{K_{\mathrm{S}}}{(4 l)^{2}}$.

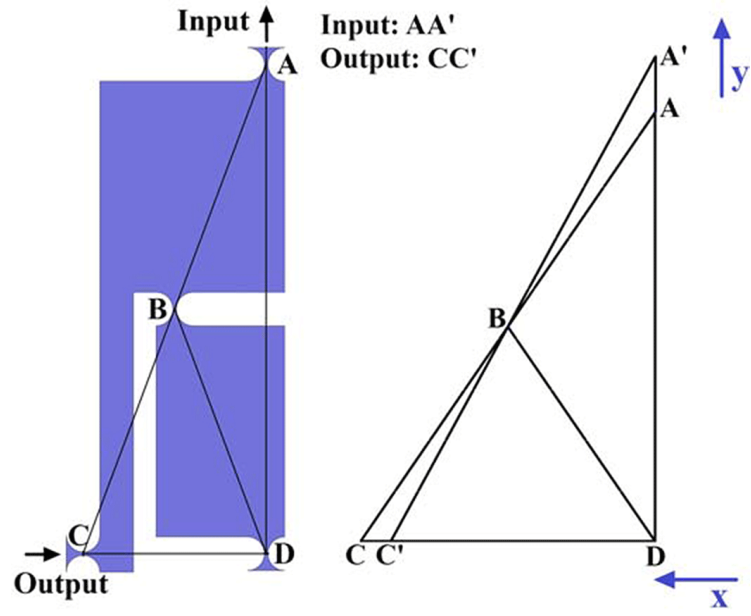

Figure 3. Schematic of Scott Russell mechanism.

\subsection{First-stage amplifier design}

The first-stage amplifier is a modified version of the SR mechanism. At first, the model of the SR mechanism will be introduced. As is known, when the lines $\mathrm{AB}=\mathrm{BC}=\mathrm{BD}$ $=L$, the structure will be consider as SR mechanism, which is shown in Fig. 3.

In order to solve the relationship between the input at point $\mathrm{A}$ and the output at point $\mathrm{C}$, assume that the displacement $\Delta y_{\mathrm{A}}$ is close to zero after input. Then, in the right triangle, one has the relationship (11). By solving the trigonometric relationship, the results of output displacement and the amplification ratio can be obtained as shown in Eqs. (12) and (13):

$$
\begin{aligned}
& \left(\Delta y_{\mathrm{A}}+l_{\mathrm{AD}}\right)^{2}+\left(\Delta x_{\mathrm{c}}+l_{\mathrm{CD}}\right)^{2} \approx\left(l_{\mathrm{AD}}^{2}+l_{\mathrm{CD}}^{2}\right), \\
& \Delta x_{\mathrm{C}}=\left(l_{\mathrm{DC}}^{2}-2 L_{\mathrm{AD}} \Delta y_{\mathrm{A}}-\Delta y_{\mathrm{A}}^{2}\right)^{\frac{1}{2}}-l_{\mathrm{DC}}, \\
& R_{1} \approx \frac{-\left[(2 L)^{2}-l_{\mathrm{AD}}^{2}\right]^{\frac{1}{2}}}{l_{\mathrm{AD}}}=-\tan \alpha,
\end{aligned}
$$

where $L=38.84 \mathrm{~mm}$ and $l_{\mathrm{AD}}=72.75 \mathrm{~mm}$ in this work.

From the above results of the amplification ratio (13), it is seen that the output of SR mechanism is decreased when the length of segment $\mathrm{CD}$ is smaller than that of $\mathrm{AD}$. It means that $\tan \alpha$ is always less than one. Hence, although SR mechanism can provide a rectilinear output, the amplification ratio is less than one. This is the disadvantage of the conventional SR mechanism. The angle $\alpha$ is set as $20.47^{\circ}$ in this work. With this setting, the amplification ratio of the SR mechanism is calculated as follows.

$$
R_{1} \approx \frac{-\left[(2 L)^{2}-l_{\mathrm{AD}}^{2}\right]^{\frac{1}{2}}}{l_{\mathrm{AD}}}=-\tan (20.47)=-0.37
$$

The minus sign means the negative direction of the output displacement. 


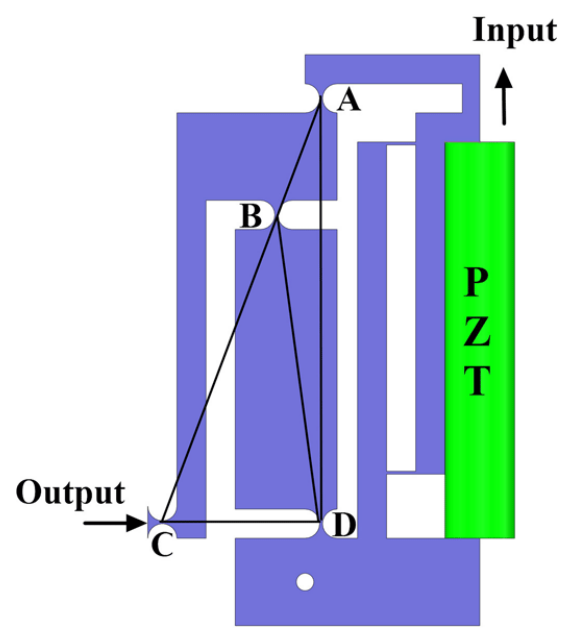

Figure 4. Modified version of Scott Russell mechanism.

In the modified SR mechanism design, the ratio between $\mathrm{AB}$ and $\mathrm{BC}$ is different. This setup not only improves the amplification ratio of the SR mechanism, but also produces approximately rectilinear output. The new amplification ratio can be calculated as follows:

$R_{2} \approx \frac{\left(l_{\mathrm{AB}}+l_{\mathrm{BC}}\right) \tan \alpha}{l_{\mathrm{AB}}}$.

A CAD model of this modified amplifier and its main parameters are shown in Fig. 4. The input from the PZT actuator is applied at the point $\mathrm{A}$ in the $y$ axis direction. The new amplification ratio can be calculated as follows:

$R_{2} \approx \frac{(22.01+55.89) \tan 20.4}{22.01}=1.32$,

where $L_{\mathrm{AB}}=22.01 \mathrm{~mm}$ and $l_{\mathrm{BC}}=55.89 \mathrm{~mm}$.

The ration $\eta$ between the amplification ratios of the new design and conventional SR mechanisms is obtained as follows.

$\eta=\frac{R_{2}}{R_{1}} \approx \frac{\left(l_{\mathrm{AB}}+l_{\mathrm{BC}}\right)}{l_{\mathrm{AB}}}$

That is,

$\eta=\frac{1.32}{0.37} \approx \frac{(22.01+55.89)}{22.01}=3.56$,

which indicates that the amplification ratio has been enlarged by 3.56 times with the new SR mechanism.

\subsection{Second-stage amplifier design}

The second-stage amplifier is a parallelogram mechanism with leaf flexures. After the first stage of amplification, the ratio is not large enough. So, the main objective of this module as an essential device is to further enlarge the output twice and to resist the nonworking directional displacement. The
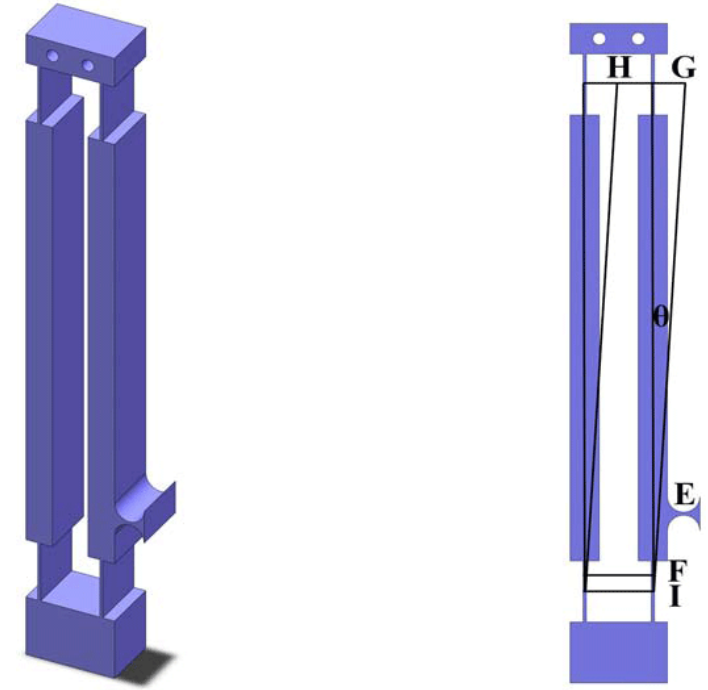

Figure 5. Model of the second-stage amplifier.

structure of this module and its main parameters are illustrated in Fig. 5. By combining the two amplifiers together, the performance of this microgripper can be further improved. The final amplification ratio of the gripper can be calculated by:

$R_{3} \approx \frac{\left(l_{\mathrm{AB}}+l_{\mathrm{BC}}\right) l_{\mathrm{IG}} \tan \alpha}{l_{\mathrm{AB}} l y_{\mathrm{IE}}}$

That is,

$R_{3} \approx \frac{(22.01+55.89) \times 83 \times \tan (20.4)}{22.01 \times 12.75}=8.59$,

which means that the amplification ratio $R_{3}$ is 8.59 for one gripper arm. During the gripping operation, the left and right arms of the gripper move together. Considering that there is only one actuator in the gripper device, the input has been amplified by 17.18 times.

Referring to Fig. 5, the rotational stiffness of point I can be calculated by (Ai and Xu, 2014):

$K_{\mathrm{r}}=\left(\frac{F_{y} l_{\mathrm{HG}}}{4+\frac{l_{\mathrm{FG}}^{2}}{l_{\mathrm{IG}}^{2}}}\right)^{\frac{1}{2}}$.

The input stiffness can be calculated as:

$K=\frac{K_{\mathrm{r}}+\frac{K_{\mathrm{r}}}{l_{\mathrm{IG}}^{2}}}{l_{\mathrm{HG}}^{2}}$.

Moreover, the equivalent mass of the gripper is shown below:

$M=M_{1}+\frac{9}{5} m_{2}+\frac{18}{125} m_{3}+\frac{1287}{25} m_{4}+\frac{4797}{40} m_{5}$, 


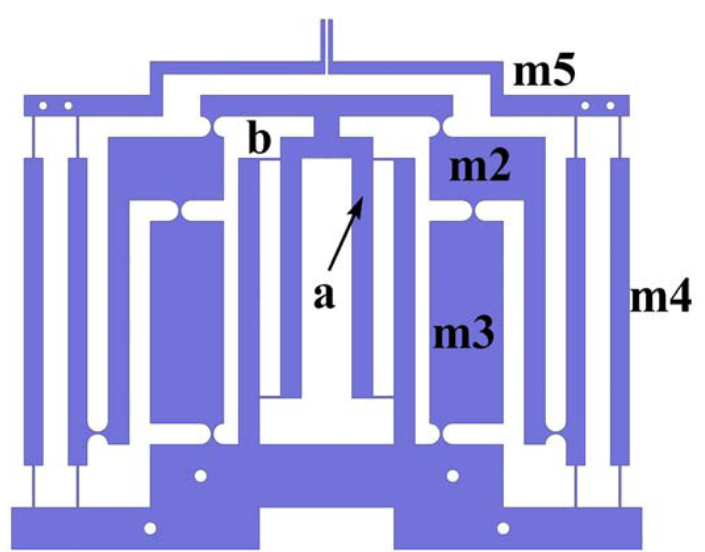

Figure 6. Mass distribution of the gripper.

where $M_{1}$ is calculated from Eq. (6) and the mass distribution of the gripper is illustrated in Fig. 6.

Then, the natural frequency in unit of Hertz can be calculated by

$f=\frac{1}{2 \pi} \sqrt{\frac{K}{M}}$.

\section{Finite-element analysis simulation}

In order to verify the analytical models as developed in the previous subsection, finite-element analysis (FEA) simulation is carried out with ANSYS to test the performance of the gripper. The simulation study includes the output displacement, nonworking directional displacement, and modal analysis. The selected material is aluminium 7075, which is known to have good mechanical properties like resistance to stress corrosion cracking and high strength in low temperature. The properties of the material are listed in Table 1. The simulation study is necessary to confirm the performance before the manufacturing of the prototype.

As shown in Fig. 7a, the maximum output displacement of the gripper is $364.4 \mu \mathrm{m}$ when the input displacement for the gripper is assigned as $45 \mu \mathrm{m}$, which is the maximum output of the PZT actuator as used in the developed prototype. In addition, it is derived from Fig. 7b that the output displacement in the nonworking direction is only $61.2 \mathrm{~nm}$, which is negligible in comparison with the output displacement in the working direction.

Moreover, modal analysis simulation has been conducted to examine the dynamic performance of the gripper structure in terms of resonant mode shapes and frequencies of the gripper structure. The simulation results of the first six resonant mode shapes are shown in Fig. 8, and the corresponding resonant frequencies are described in Table 2. It is seen that the first frequency of the gripper is $70.7 \mathrm{~Hz}$. A high natural frequency enables a quick response of the gripper device. In
Table 1. Properties of aluminium 7075 alloy.

\begin{tabular}{lcc}
\hline Item & Value & Units \\
\hline Elongation & $11 \%$ & - \\
Density & 2810 & $\mathrm{~kg} \mathrm{~m}^{-3}$ \\
Modulus of elasticity & 72 & $\mathrm{GPa}$ \\
Yield strength & 455 & $\mathrm{MPa}$ \\
Tensile strength & 524 & $\mathrm{MPa}$ \\
Poisson ratio & 0.33 & - \\
\hline
\end{tabular}

Table 2. The first-six resonant frequencies.

\begin{tabular}{cc}
\hline Mode & Frequency $(\mathrm{Hz})$ \\
\hline 1 & 71.715 \\
2 & 77.338 \\
3 & 148.82 \\
4 & 149.84 \\
5 & 440.55 \\
6 & 443.31 \\
\hline
\end{tabular}

Table 3. Specification of the PZT actuator.

\begin{tabular}{lcc}
\hline Item & Value & Units \\
\hline Model & P840.3 & - \\
Open loop travel & 45 & $\mu \mathrm{m}$ \\
Pushing force & 1000 & $\mathrm{~N}$ \\
Pulling force & 50 & $\mathrm{~N}$ \\
Outer length & 12 & $\mathrm{~mm}$ \\
Length & 68 & $\mathrm{~mm}$ \\
Resolution (open loop) & $0.9(0.45)$ & $\mathrm{nm}$ \\
\hline
\end{tabular}

addition, modes 3 and 4 are caused by the vertical translations in the $y$ axis, and modes 5 and 6 are induced by the bending of the gripper arm around the $z$ axis.

From the simulation results, it is observed that the objective of design has been achieved. This microgripper can provide both a large-range output and an approximately rectilinear movement. The rectilinear movement is useful for gripping various micro-particles in practice.

\section{Experimental results}

After the FEA simulation verification, the device has been manufactured. Then, an experimental study is conducted to test the performance of the prototype. The aim of the experiment is to verify the output displacement of the gripper. The experimental setup is depicted in Fig. 9. A laser sensor is used to detect the output displacement of the gripper tip. The sensor has a resolution about $25 \mathrm{~nm}$. A PZT actuator is used to actuate the gripper and its specifications are listed in Table 3. Moreover, the actuator has a working voltage range of 0-100 V. 
(a)

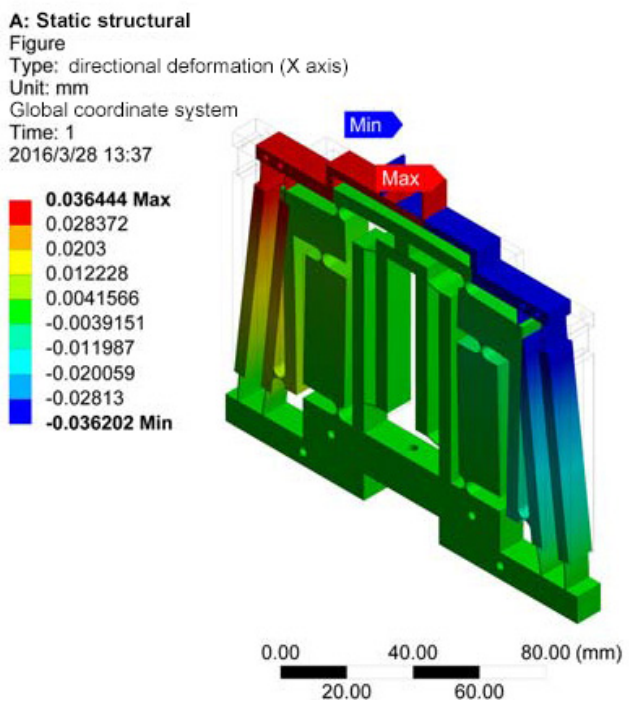

(b)

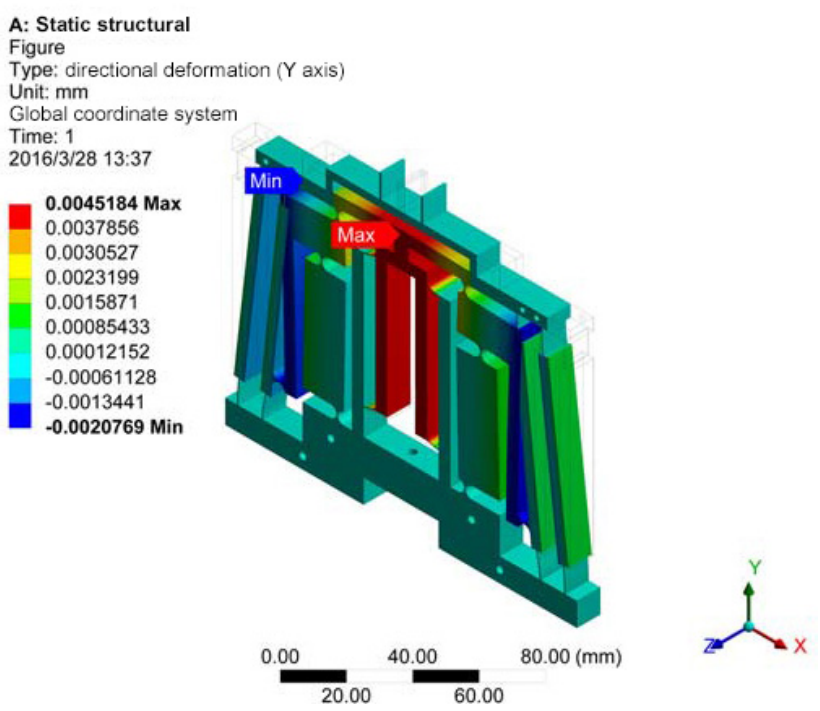

Figure 7. FEA simulation results. (a) Maximum output of the gripper, (b) displacement in nonworking direction.

It is observed that when the driving voltage approaches to $100 \mathrm{~V}$, the output displacement arrives at the largest value. The results collected by the laser sensor are shown in Table 4. The open and close states of the gripper tips are illustrated in Fig. 10. Moreover, for the purpose of comparison, the analytical model result, FEA simulation result, and experimental result are listed in Table 5. It is observed from Table 5 that the experimental result is very close to the analytical and simulation results, which verifies the effectiveness of the developed models.

The results of the conducted experimental studies verify the design of this new large-range compliant microgripper. A modified version of SR mechanism is proposed to enhance the performance of the original version. The objective is to enlarge the amplification ratio and to resist the nonworking directional displacement by using one more parallelogram module. The FEA simulation shows that the nonworking directional displacement is $61.2 \mathrm{~nm}$. This performance can be optimized by an optimum design to be conducted in the future work.

In order to obtain a larger gripping range in micromanipulation, the dual-stage amplifier provides an amplification ratio of 8.066 per arm. Totally, the gripping range is enlarged to be 16.13 times of the driving displacement, which is enabled by the proposed new gripper design with modified SR amplification mechanism. As compared with the existing grippers, in which only one arm is actuated, the two-arm actuation approach with dual-stage amplification mechanism produces a much larger output gripping range.

For practical applications, the grasp force exerted on the object should lie in an appropriate range (Liu et al., 2009; $\mathrm{Xu}, 2015)$. Hence, the grasp force regulation is necessary to guarantee that an appropriate force is applied to the grasped
Table 4. Experimental results of output displacement of the compliant microgripper.

\begin{tabular}{lcccc}
\hline Item & Test 1 & Test 2 & Test 3 & Test 4 \\
\hline $\begin{array}{l}\text { Output displacement } \\
\text { of one gripper arm }(\mu \mathrm{m})\end{array}$ & 362 & 363 & 360 & 363 \\
\hline
\end{tabular}

Table 5. Comparison of the results.

\begin{tabular}{lccc}
\hline Item & Test 1 & Test 2 & Test 3 \\
\hline $\begin{array}{l}\text { Output displacement } \\
\text { of one gripper arm }(\mu \mathrm{m})\end{array}$ & 362 & 363 & 360 \\
Error & $7.2 \%$ & $0.88 \%$ & - \\
\hline
\end{tabular}

objects (Komati et al., 2013; Xu, 2015). In the future work, the force sensing and control system module will be realized to deliver a desired output force of the gripper for executing related micromanipulation tasks. Pertinent application study will be carried out as well.

\section{Conclusions}

In this paper, a new design of large-displacement gripper is analyzed, fabricated, and tested. Each module of the gripper is designed in detail. After the analytical model has been developed, simulation study has been conducted to verify the performance of the design. Finally, a prototype of the gripper has been manufactured for experimental testing. Results show that the gripping range is $0-720 \mu \mathrm{m}$ along with a large displacement amplification of 16.13. It provides enough output motion for the manipulation of majority of micro- 
(a)

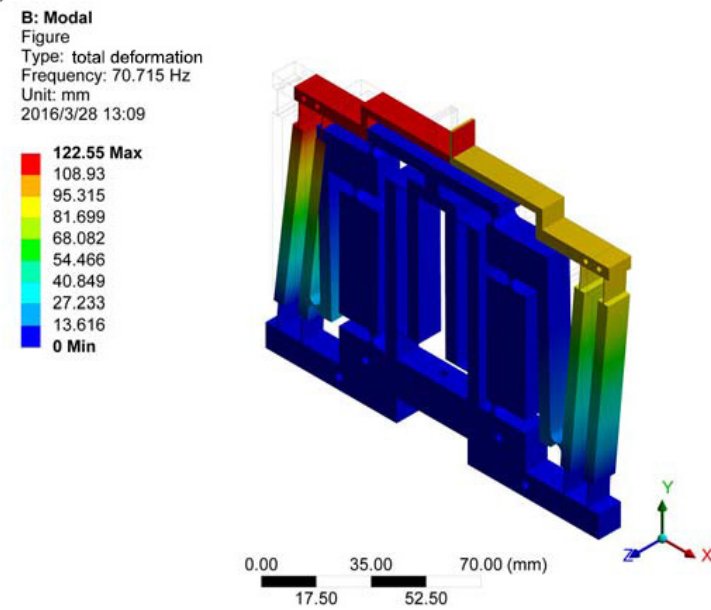

(c)

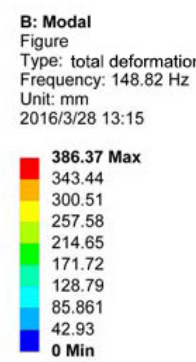

Figure Frequency: $148.82 \mathrm{~Hz}$ 2016/3/28 13:15

(e)
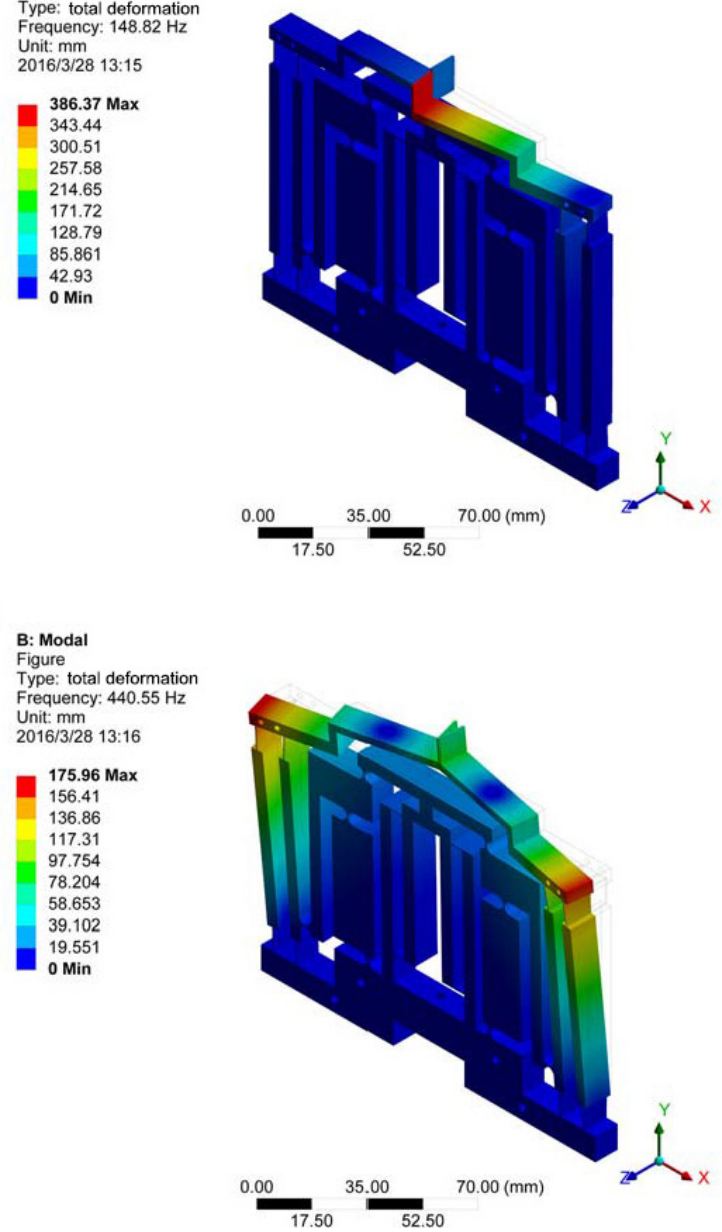

(b)

B: Modal

Type: total deformatio Frequency: $77.338 \mathrm{~Hz}$

$122.29 \mathrm{Max}$

108.7

95.111

81.523
67.936

67.936
54.349

40.762

27.174

13.587

0 Min Unit: $\mathrm{mm}$ 2016/3/28 13:15

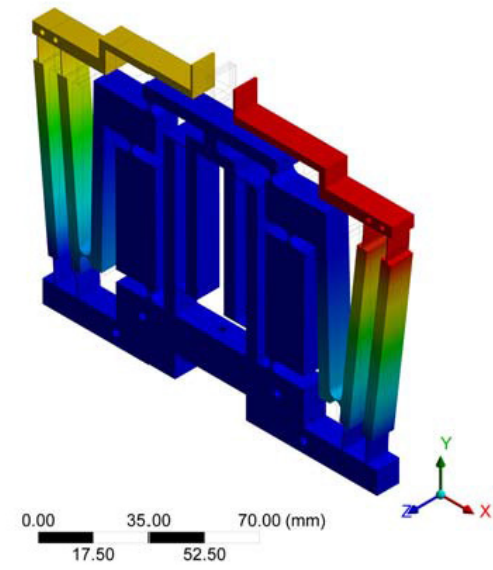

(d)

(f)
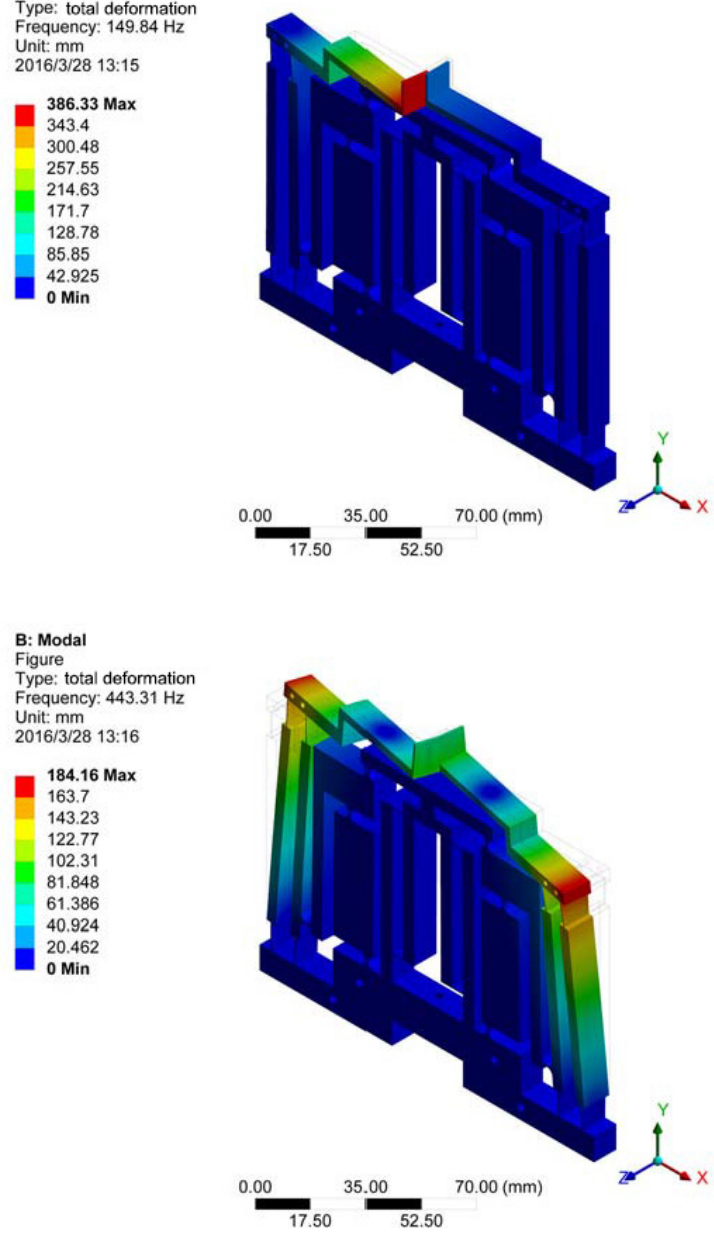

B: Modal
Figure

Type: total deformation Frequency: 149.84 2016/3/28 13:15

$\mathbf{3 8 6 . 3 3}$ Max
343.4
300.48
257.55
214.63
171.7
128.78
85.85
42.925
0 Min

Figure 8. The first six resonant mode shapes (a-f).

particles. The amplification ratio of the modified SR mechanism has been enlarged by 3.56 times relative to the traditional design. In the future work, a precision motion and

force control module will be implemented on the developed gripper to produce a desired output force dedicated to related micromanipulation tasks. 


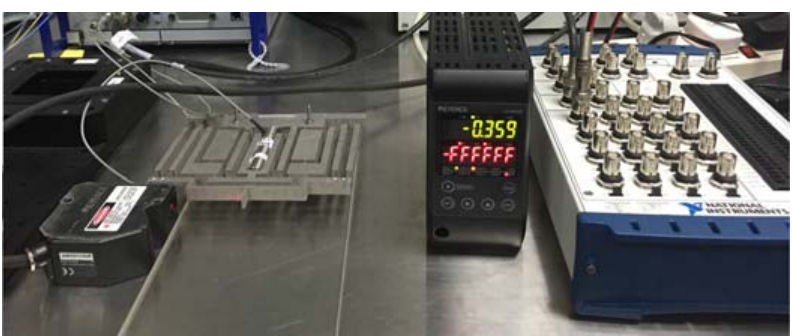

Figure 9. Experimental setup of the microgripper.

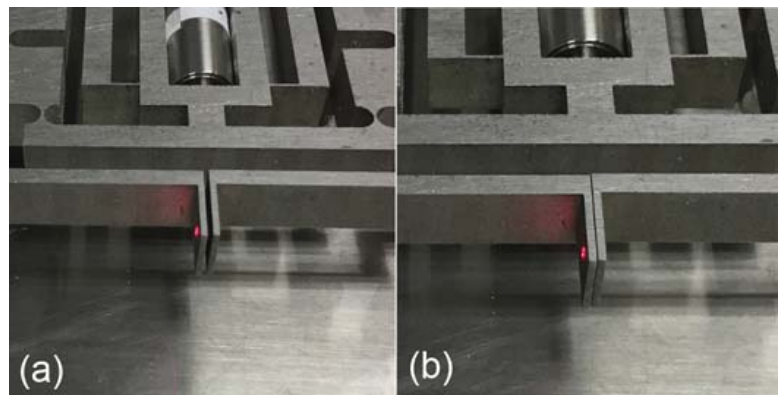

Figure 10. Photos of the gripper tips before (a) and after (b) the actuation.

Acknowledgements. This work was supported in part by the Macao Science and Technology Development Fund under Grant 090/2015/A3 and 052/2014/A1, and the Research Committee of the University of Macau under Grant MYRG078(Y1-L2)-FST13-XQS.

Edited by: K. Mianowski

Reviewed by: L. Bruzzone and M. Brandstötter

\section{References}

Ai, W. and Xu, Q.: New structural design of a compliant gripper based on the Scott-Russell mechanism, Int. J. Adv. Robot. Syst., 11, doi:10.5772/59655, 2014.

Arai, F. and Fukuda, T.: Adhesion-type micro end effector for micromanipulation, in: Proceedings of 1997 IEEE International Conference on Robotics and Automation, Albuquerque, NM, 20-25 April 1997, IEEE, 2, 1472-1477, 1997.

Bergander, A., Driesen, W., Varidel, T., and Breguet, J.-M.: Development of miniature manipulators for applications in biology and nanotechnologies, in: Proc. of IEEE/RSJ Int. Conf. on Intelligent Robots and Systems (IROS 2003), Las Vegas, NV, USA, 27-31 October 2003, 11-35, 2003.

Bruzzone, L. and Bozzini, G.: A flexible joints microassembly robot with metamorphic gripper, Assembly Autom., 30, 240247, 2010.

Chang, S. and Du, B.: A precision piezodriven micropositioner mechanism with large travel range, Rev. Sci. Instrum., 69, 17851791, 1998.
Dunning, A. G., Tolou, N., and Herder, J. L.: Review Article: Inventory of platforms towards the design of a statically balanced six degrees of freedom compliant precision stage, Mech. Sci., 2, 157-168, doi:10.5194/ms-2-157-2011, 2011.

Götze, H. and Pagel, L.: Development of a micro-manipulator based on piezoelectric-technology, Microelectron. Eng., 84, 13331336, 2007.

Ha, J.-L., Kung, Y.-S., Hu, S.-C., and Fung, R.-F.: Optimal design of a micro-positioning Scott-Russell mechanism by Taguchi method, Sensor. Actuat. A-Phys., 125, 565-572, 2006.

Han, K., Lee, S. H., Moon, W., and Park, J.-S.: Fabrication of the micro-gripper with a force sensor for manipulating a cell, in: Proc. of SICE-ICASE International Joint Conference, IEEE, Bexco, Busan, Korea, 5833-5836, 2006.

Hwang, D., Byun, J., Jeong, J., and Lee, M. G.: Robust design and performance verification of an in-plane $\mathrm{XY} \theta$ micropositioning stage, IEEE T. Nanotechnol., 10, 1412-1423, 2011.

Khadraoui, S., Rakotondrabe, M., and Lutz, P.: Optimal design of piezoelectric cantilevered actuators with guaranteed performances by using interval techniques, IEEE-ASME T. Mech., 19, 1660-1668, 2014.

Komati, B., Rabenorosoa, K., Clévy, C., and Lutz, P.: Automated guiding task of a flexible micropart using a two-sensing-finger microgripper, IEEE T. Autom. Sci. Eng., 10, 515-524, 2013.

Lamers, A., Sánchez, J. A. G., and Herder, J. L.: Design of a statically balanced fully compliant grasper, Mech. Mach. Theory, 92, 230-239, 2015.

Liu, X., Kim, K., Zhang, Y., and Sun, Y.: Nanonewton force sensing and control in microrobotic cell manipulation, Int. J. Robot. Res., 28, 1065-1076, 2009.

Merken, P., Smal, O., Debongnie, J.-F., and Raucent, B.: Design and test of a circular notch hinge, in: Proceedings of the International Precision Assembly Seminar (IPAS 2004), Bad Hofgastein, Austria, 51-56, 2004.

Merriam, E. G., Jones, J. E., Magleby, S. P., and Howell, L. L.: Monolithic 2 DOF fully compliant space pointing mechanism, Mech. Sci., 4, 381-390, doi:10.5194/ms-4-381-2013, 2013.

Tanikawa, T., Arai, T., and Masuda, T.: Development of micro manipulation system with two-finger micro hand, in: Proceedings of IEEE/RSJ International Conference on Intelligent Robots and Systems (IROS 96), IEEE, Osaka, Japan, 2, 850-855, 1996.

Tian, Y., Liu, C., Liu, X., Wang, F., Li, X., Qin, Y., Zhang, D., and Shirinzadeh, B.: Design, modelling and characterization of a 2-DOF precision positioning platform, T. I. Meas. Control, 37, 396-405, 2015.

$\mathrm{Xu}, \mathrm{Q}$.: Design, testing and precision control of a novel long-stroke flexure micropositioning system, Mech. Mach. Theory, 70, 209224, 2013.

$\mathrm{Xu}, \mathrm{Q} .:$ Design and development of a novel compliant gripper with integrated position and grasping/interaction force sensing, IEEE T. Autom. Sci. Eng., doi:10.1109/TASE.2015.2469108, online first, 2015.

Zhang, Y., Lu, T.-F., and Al-Sarawi, S.: Formulation of a simple distributed-parameter model of multilayer piezoelectric actuators, J. Intel. Mat. Syst. Str., doi:10.1177/1045389X15595294, online first, 2015. 